\title{
Medial Prefrontal Theta Bursts Precede Rapid Motor Responses during Visual Selective Attention
}

\author{
Arnaud Delorme, ${ }^{1}$ Marissa Westerfield, ${ }^{2}$ and Scott Makeig ${ }^{1}$ \\ ${ }^{1}$ Swartz Center for Computational Neuroscience, and ${ }^{2}$ Department of Neuroscience, University of California San Diego, La Jolla, California 92093
}

\begin{abstract}
After visual target stimuli presented infrequently at a covertly attended location, quicker speeded button presses immediately followed a larger positive (P3f) ramp in averaged electroencephalographic (EEG) recordings from the forehead. We show this peak in the mean response time locked to the button press to be principally composed of triphasic, primarily low-theta band $(4.5 \mathrm{~Hz})$ complexes preceding but only partially phase-locked to the button press, with larger complexes preceding quicker motor responses. For 10 of 15 subjects, independent component analysis of the unaveraged 31-channel data identified a temporally independent medial frontal EEG process contributing to these phenomena. Low-resolution tomographic modeling localized related components of two 253-channel data sets to medial frontal polar cortex (BA32/10). The far-frontal low-theta complexes and concomitant mean P3f positivity may index cortical activity induced by paralimbic processes involved in disinhibiting impulsive motor responses to rewarding or goal-fulfilling stimuli or events.
\end{abstract}

Key words: theta; reaction time; VMPFC; EEG; ICA; medial prefrontal cortex

\section{Introduction}

During the development of the field of experimental psychology, measurements of response times (RTs), the ubiquitous dependent variable of psychology, were long a primary tool for inferring mental organization (Luce, 1986). The early psychologist Wilhelm Wundt hypothesized that faster responders react more reflexively to response cues, whereas slower responders wait for a more fully elaborated conscious perception of the cue stimulus (Wundt, 1913). This hypothesis has been little explored physiologically. Voluntary motor response decisions are usually thought to be triggered primarily in frontal "executive" brain areas, including orbitofrontal cortex (Liu et al., 2004), medial prefrontal cortex, and posterior, central, and dorsal anterior cingulate cortex (Naito et al., 2000; Mesulam et al., 2001; Ullsperger and von Cramon, 2003). Although a few functional magnetic resonance imaging (fMRI) studies have reported correlations between single-trial blood-oxygen level dependant (BOLD) signal changes and RTs in frontopolar and anterior cingulate cortex (Naito et al., 2000; Mesulam et al., 2001; Liu et al., 2004), the slow time course and poor time resolution of BOLD signals makes it difficult to determine whether the concerned brain activity precedes or follows the motor response.

Received May 9, 2006; revised Feb. 27, 2007; accepted Sept. 3, 2007.

This work was supported by the Swartz Foundation (Old Field, NY), National Institutes of Health Grant R01NS047293, and the long-time collaboration of Jeanne Townsend (under whose project most of the data were collected) and Eric Courchesne (in whose laboratory they were recorded). We thank Julie Onton for help with the 256-channel data acquisition and Stefan Debener, Guillaume Rousselet, and an anonymous reviewer for providing helpful comments on this manuscript.

Correspondence should be addressed to Arnaud Delorme, Swartz Center for Computational Neuroscience, Institute for Neural Computation, University of California San Diego, La Jolla, CA 92093-0961. E-mail: arno@sccn.ucsd.edu.

DOI:10.1523/JNEUROSCI.3477-07.2007

Copyright $\odot 2007$ Society for Neuroscience 0270-6474/07/2711949-11\$15.00/0
Scalp electroencephalographic (EEG) recordings, in contrast, give noninvasive, millisecond-scale temporal information about the macroscopic dynamics of human cortical activity, making EEG recordings suited to identifying frontal brain activity linked to RTs. In monkeys and humans, RTs have been found to be correlated with differences in gamma power and gamma coherence (Jokeit and Makeig, 1994; Gonzalez Andino et al., 2005; Kilner et al., 2005; Schoffelen et al., 2005; Womelsdorf et al., 2006) but not at lower frequencies. Scalp event-related potentials (ERPs) are composed principally of these lower frequencies, but thus far, RTs have only been found to be correlated with ERP features that follow rather than precede the motor response (Hohnsbein et al., 1991; Proverbio et al., 1994; Bahramali et al., 1998; Tenke et al., 1998; Travis, 1998; Kida et al., 2003). Searching for correlations between RTs and average ERP features, however, fails to address the complexity of the effect of stimulation and generation of behavioral responses on ongoing cortical activities in the single trials (Makeig et al., 2002), which may exhibit event-related changes as early as $100 \mathrm{~ms}$ after visual stimuli (Klopp et al., 2000).

Here, we used independent component analysis (ICA) to analyze EEG data from a visual "oddball" paradigm in which participants were asked to respond quickly to rare targets presented semirandomly among a stream of nontarget stimuli. First, we confirm that the amplitude of the early far-frontal peak of the response-locked ERP in single trials of these data, termed P3f by Makeig et al. (1999b, 2004b), was larger just before faster motor responses. Next, we show this P3f peak is almost entirely accountable as a phase-varying, typically triphasic, low-theta band complex preceding but not precisely time locked to speeded button presses. Finally, we use results of low-resolution electrical tomography to suggest that the far-frontal P3f peak and underlying theta-band complexes may primarily arise in medial prefrontal cortex. 


\section{Materials and Methods}

Task design. Fifteen right-handed subjects (age range, 19-53 years; mean 30 years) with normal or corrected to normal vision participated in the experiment. Filled white disks appeared briefly inside one of five empty squares that were constantly displayed $0.8 \mathrm{~cm}$ above a central fixation cross following the design of Townsend et al. (1996). The $1.6 \mathrm{~cm}$ square outlines were displayed in blue on a black background at horizontal visual angles of $0 \pm 2.7$ and $0 \pm 5.5^{\circ}$ from fixation. During each $76 \mathrm{~s}$ block of trials, one of the five outlines was colored green, marking the location to be attended. The subject made a motor response by pressing a mouse button with their right hand as quickly as possible whenever the filled white disk ( $\sim 1.4 \mathrm{~cm}$ in diameter) appeared at the attended location. This location was counterbalanced across blocks in pseudorandom order.

In each block, 100 stimuli (filled white disks) were displayed for $117 \mathrm{~ms}$ within one of the five empty squares in a pseudorandom sequence with interstimulus intervals (ISIs) of 250-1000 ms (in four equiprobable 250 ms steps). Thirty blocks of trials were collected from each subject, yielding 120 target and 480 nontarget trials at each location. Subjects were given breaks between blocks. In the present report, we consider only the correct target response trials in which subjects responded 150-1000 ms after target onset (which amount to $>95 \%$ of all target trials). Average stimulus-locked ERP and single-trial analyses of both early (N1) and late target-related activity in these data have been reported previously (Makeig et al., 1999a,b, 2002, 2004b).

EEG recordings. EEG data were collected from 29 scalp electrodes mounted in a standard electrode cap (Electrocap) at locations based on a modified International 10-20 System and from two periocular electrodes placed below the right eye and at the left outer canthus. All channels, including suborbital channels, were referenced to the right mastoid. Input impedance was brought below $5 \mathrm{k} \Omega$ for all electrodes. Data were sampled at $512 \mathrm{~Hz}$ with an analog pass band of 0.01-50 Hz. Data were down sampled to $256 \mathrm{~Hz}$, and, to further minimize line noise artifacts responses, were digitally low-pass filtered below $40 \mathrm{~Hz}$ before data analysis. Data trials were originally extracted using a $3 \mathrm{~s}$ time window starting $1 \mathrm{~s}$ before stimulus onset. Data trials containing electrooculographic (EOG) potentials larger than $70 \mu \mathrm{V}$ were rejected.

To better explore the location of the brain source responsible for the effect we report here, we also recorded 253-channel data from two subjects with a $256 \mathrm{~Hz}$ sampling rate using a Biosemi Active Two system. We preprocessed and pruned these data of artifacts as described above.

Power/RT correlations in single trials. We computed correlations between single-trial subject RT and response-locked EEG power at a matrix of time-frequency points. Before correlating RT with spectral power, spectral phase, or ERP amplitude, we removed the 5\% fastest and the 5\% slowest trials to reduce the influence of extreme RT values. To compute EEG spectral power, we convolved the single-trial data activity from a scalp electrode (or independent component) with a three-cycle complex Morlet wavelet

$$
\psi_{f}(k)
$$

(Grossman and Morlet, 1985). At a given time $t$ and frequency $f$ :

$$
S_{e}(f, t)=\sum_{k=1}^{N} d_{e}\left(t+\frac{k-N / 2-1}{s}\right) \psi_{f}(k)
$$

with

$$
\left|S_{e}(f, t)\right|
$$

the spectral amplitude estimate from electrode $e$ and $d_{e}(t)$ the potential of electrode $e$ at time $t$. Here, $k$ is the index of the bin from 1 to $N$, and $s$ is the signal sampling rate. At each time $t$ and frequency $f$, we correlated RT with EEG power

$$
P_{e}(f, t)=10 \log _{10}\left(\left|S_{e}(F, t)\right|^{2}\right) .
$$

We then generated two-dimensional (2-D) correlation images in which each pixel represented standard linear regression (Draper and Smith, 1981) of time-frequency power estimate for all data trials and all subjects
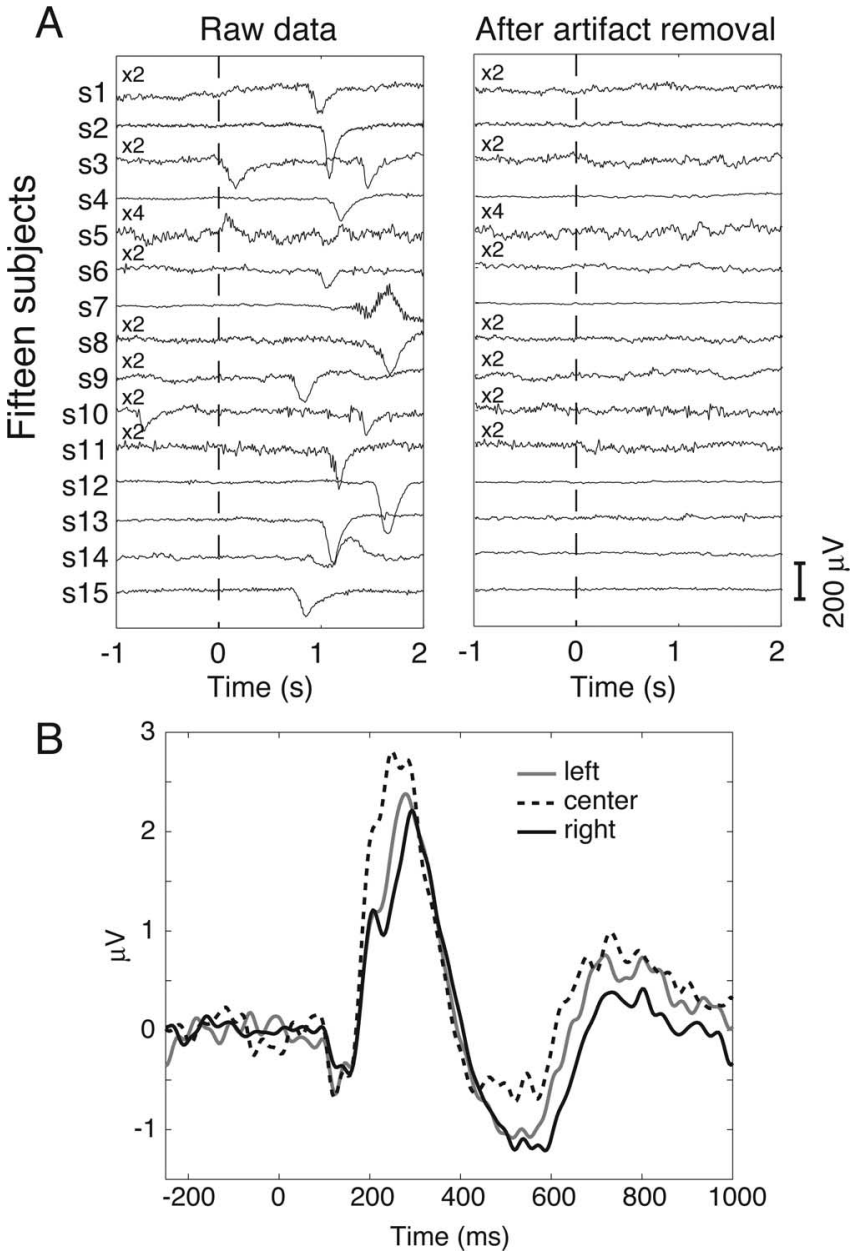

Figure 1. A, Artifact removal from single trials recorded at a right subocular electrode from 15 subjects (s1-s15). Left, Sample data from each subject including eye blink artifacts of varied shapes and amplitudes. Time 0 indicates the presentation of the stimulus. Some traces are shown magnified (twice, $\times 2$; or four times, $\times 4$ ) for easier visualization. Right, The same trials after artifact removal of six clusters of independent components accounting for nonbrain artifacts including eye movements. B, Grand-average stimulus locked ERPs for the periocular channel after removal of artifact components, as a function of target location (above left, directly above, or above right) relative to subject eye fixation. The P3f positivity near $300 \mathrm{~ms}$ is nearly identical in response to left and right targets. If lateral eye movements were contributing to the response, the left and right target responses might be expected to differ; after artifact removal, they do not.

on RTs in the same trials. The 2-D correlation images contained 58 frequencies equally log spaced through the frequency range $2.5-50 \mathrm{~Hz}$, and 81 time points regularly spaced in the latency range -200 to $750 \mathrm{~ms}$ relative to the median stimulus onset latency. The number of wavelet cycles increased linearly across log-frequencies, from $3($ at $2.5 \mathrm{~Hz})$ to 18 (at $50 \mathrm{~Hz}$ ). We also computed linear regressions between single-trial log spectral power and relative RT (removing each subject's median RT from all their trial RTs). All 2-D correlation images were masked for significant effects at $p<0.0001$. Because of strong correlations of power estimates within a time-frequency neighborhood, we estimated the number of independent comparisons in the 2-D correlation images as 100 (Tanji et al., 2005), so $p=0.0001$ (uncorrected) corresponded to $p=0.01$ after correction for multiple comparisons (Bonferroni, 1950). [An alternative way to correct for multiple comparisons would have been to use the method developed by Nichols and Holmes (2002)].

Independent component analysis. Infomax ICA (Bell and Sejnowski, 1995; Makeig et al., 1996) is one of a family of algorithms that exploit temporal independence to perform blind separation. Lee et al. (2000) have shown that these algorithms have a common information theoretic basis. Infomax ICA finds, by natural gradient ascent, a square "unmix- 

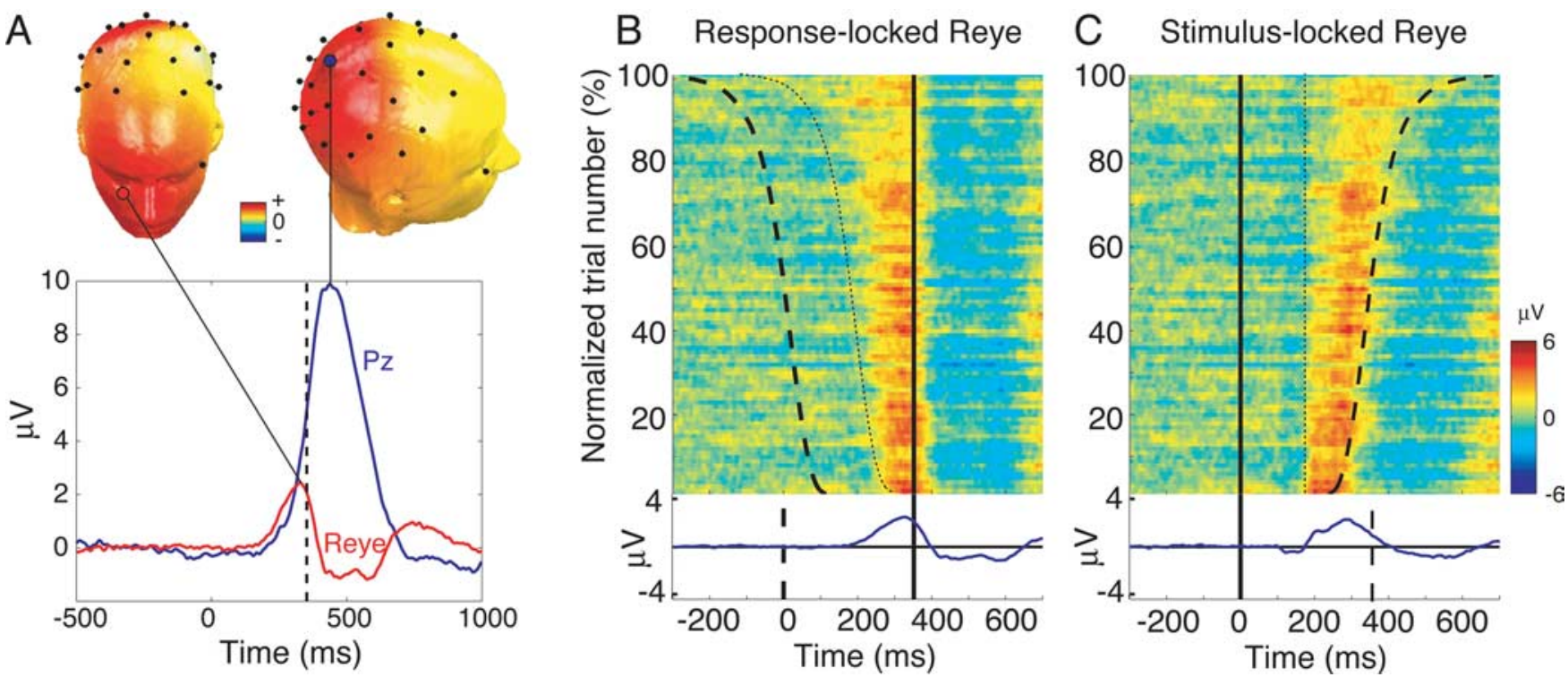

Figure 2. A, Artifact-corrected grand-average motor response-locked ERP from 15 subjects at two scalp channels: right subocular (left) and central parietal ( $P z$; right). A positive ERP peak at the subocular channel (P3f) precedes the motor response (vertical line), whereas the larger late positive (P3) peak over parietal cortex follows the motor response by $\sim 150$ ms. Head cartoons represent the ERP scalp topography at the two peak response latencies. $\boldsymbol{B}$, Event-related activity at the right suborbital channel in grand-average ERP-image format. Here, the dashed trace indicates the moment of the motor response. For each subject, single data trials aligned to the button press (solid vertical line) were sorted by reaction time after stimulus onset (dashed trace) and then smoothed (vertically) using a 10-trial moving subaverage. The trial subaverages were then averaged across subjects and visualized as horizontal color-coded lines. $\boldsymbol{C}$, Same data epochs as in $\boldsymbol{B}$, here, time locked to stimulus onset (solid line). In $\boldsymbol{B}$ and $\boldsymbol{C}$, thin dotted traces mark the earliest P3f onset, near $200 \mathrm{~ms}$ after stimulus onset.

ing" matrix that maximizes the joint entropy of a nonlinearly transformed ensemble of zero-mean input data vectors. Here, we used the same decomposition of target stimulus epochs used in a previous report (Makeig et al., 2004b). The runica algorithm (Makeig et al., 1997) [available for download in the EEGLAB toolbox (Delorme and Makeig, 2004) at http://sccn.ucsd.edu/eeglab], an implementation of extended infomax ICA, was applied to sets of $400-600$ single $1 \mathrm{~s}$ trials (31 channels, 256 time points) time locked from $200 \mathrm{~ms}$ before to $800 \mathrm{~ms}$ after onsets of target stimuli.

The total of $31 \times 15=465$ components for the 15 subjects were separated into clusters by applying a modified Mahalanobis distance measure to vectors coding component map gradients [ 62 gradient measures reduced to 12 dimensions using principal component analysis (PCA) ] and component activity log spectra (50 frequencies from 1 to 50 $\mathrm{Hz}$ reduced to five dimensions using PCA). Cluster membership was, in a few cases, then further adjusted by eye for uniformity.

For the 256-channel recordings, to maximize the number of data points per weight in the training data, we decomposed the continuous data with extended infomax ICA directly (not the concatenated data epochs) after reducing the data to 150 principal dimensions by PCA.

Source localization. To better localize brain areas corresponding to component scalp topographies, the digitized three-dimensional (3-D) localizations of the electrodes were first coregistered with the average MNI brain (Montreal Neurological Institute) and exported to the LORETA software (Pascual-Marqui et al., 1994). Electrodes lower than $-50 \mathrm{~mm}$ in elevation in the MNI image coordinates were excluded to minimize mislocalization from inaccuracies in the frontal skull model. After calibration using a collection of independent components, the "oversmoothness" parameter was set to $10^{-3}$ for both subjects. Gray matter voxel activity was exported and overlaid on normalized individual subject MR images using custom Matlab (The MathWorks, Natick, MA) scripts. [MR images were spatially normalized to the MNI brain using the SPM2 package (Ashburner and Friston, 2003)]. To increase contrast for the diffuse localization on the dark MRI background, a gamma factor of 3 on voxel activity was used. Component scalp map projections in time are scaled by component activities, so scalp maps themselves do not have absolute amplitudes. For this reason, the scale is not shown in Figure 9. For normalized component scalp maps, the maximum activity in the LORETA map is $\sim 1.10-4 \mathrm{Amp} / \mathrm{m}^{2}$.

\section{Results}

Analysis of periocular EEG

In most EEG research, neural activity recorded by periocular data channels is labeled EOG activity, and other EEG phenomena expressed in these data are ignored for fear of mislabeling eyeactivity artifacts as brain activity. However, some independent components of EEG signals can be clearly identified as accounting primarily for eye movements, for line or muscle noise, or for other artifacts, based on characteristics of their scalp maps and activity time courses (Makeig et al., 1996; Jung et al., 2000). Therefore, after performing ICA decomposition separately on the concatenated 31-channel data trials for each subject, we removed six clusters of independent components accounting for eye movement and muscle activity artifacts. Eye movements and other artifacts in the raw suborbital channel data (Fig. 1 A, left) were markedly reduced or eliminated after subtracting the identified artifacts (Fig. $1 \mathrm{~A}$, right).

Although the subjects were asked to fixate the center of the screen while performing this spatial attention task, it is possible that lateral saccades toward targets could have influenced the stimulus-locked ERP. To address this issue, Figure $1 B$ shows grand-average ICA-pruned, stimulus-locked ERPs at the right periocular channel for stimuli presented directly above, above right, and above left of fixation, respectively. The data support the hypothesis that the P3f peak is minimally contaminated by horizontal eye saccades: the P3f feature of the evoked responses to the left and right visual field target stimulus presentations is of lower amplitude when compared with the P3f response to centrally presented stimuli, as is also true for the central P300 peak (Neville and Lawson, 1987). Thus, ICA made it possible for us to examine EEG brain activity recorded at this periocular channel apart from artifacts produced by eye movements.

\section{Premovement positivity}

The bulk of the $\mathrm{P} 3$ peak in the average ERP response to targets occurred after the subject response (Fig. $2 \mathrm{~A}$ ). However, at the 
right subocular channel, a positive ERP peak preceded the motor response. Figure $2 \mathrm{~B}$ shows ERP-image plots (Makeig et al., 1999b) of trial data from the right suborbital channel after pruning for artifacts. In these plots, potential changes across time in single trials (or in local averages of a few trials) are represented by successive thin, color-coded horizontal lines of a twodimensional image. To construct these grand-mean ERP images, individual subject ERP-image plots were first produced by sorting their response-locked trials by RT and vertically smoothing with a 10-trial moving average. The 15 subject ERP images were then averaged. Thus, in these images (Fig. $2 B, C$ ), every subject contributes equally to each moving trial average (i.e., to each color-coded horizontal line).

The positive preresponse ERP peak (P3f) was maximal at forehead sites. Comparing Figure $2 B$ and $2 C$, it is clear that the P3f peak is more time locked to the motor response than to the stimulus onset. This was tested formally by computing the peak intertrial coherence (ITC) before the button press at $4.5 \mathrm{~Hz}$, the center frequency of the P3f feature. ITC is a measure of the consistency of spectral phase of recorded activity across single trials (TallonBaudry et al., 1996; Makeig et al., 2002; Delorme and Makeig, 2004). If, at a given frequency and latency, relative to the timelocking events, ITC is close to 0 , then the measured spectral phase is evenly distributed across trials, whereas if ITC is near 1, then the activity is reliably phase locked to the time-locking events. At $4.5 \mathrm{~Hz}$ and $50 \mathrm{~ms}$ preceding the button press, the mean ITC in the response-locked trials was $0.45( \pm 0.11)$, whereas in the stimuluslocked trials at the same mean latency, it was $0.34( \pm 0.11)$. A paired $t$ test across the 15 subjects confirmed the significantly higher ITC in the response-locked trials $(\mathrm{df}=14 ; p=0.0018)$. Although we processed both stimulus-locked and responselocked trials, stimulus-locked trials did not appear to provide additional information; therefore, below, we focus on results for response-locked trials.

\section{Correlations with RT}

Figure 2, $B$ and $C$, shows that trials with shorter RTs (toward the bottom of each image) were generally preceded by a larger preresponse P3f positivity than longer-RT trials (above). To quantify this relationship between single-trial EEG activity and RT, we first computed grand-mean ERP averages of trials in six RT-range quantiles (Fig. $3 A$ ). For each subject, we partitioned the data trials into six RT ranges of equal size and then computed the mean ERP of trials in each RT range. Finally, we grand averaged the resulting subject ERPs across the 15 subjects for each RT range. In the response-locked ERP data (Fig. 3A), only the subaverage values near the preresponse P3f peak covaried with RT $(p<0.0001)$. Although nine of the 15 subjects showed a similar within-subject correlation (at $p<0.001$ ) for latencies from $150 \mathrm{~ms}$ before to 50 $\mathrm{ms}$ after the button press, the correlation between the 15 subjectmedian RTs and subject-mean P3f peak amplitudes did not reach significance. To confirm that larger P3f amplitude correlates with reaction time, we separated each subject's trials into 20 RT quantiles and averaged these across subjects. In these within-subject quantile averages, a larger preresponse P3f peak $25 \mathrm{~ms}$ before the button press preceded faster RTs $(p<0.001)$.

As shown in Figure $3 B$, there was also a very strong linear relationship $\left(r^{2}=0.93 ; p<10^{-11}\right)$ between quantile-median RT and the upward slope of the rising P3f peak (computed by linear regression on the subaverage waveforms from 150 to $25 \mathrm{~ms}$ before the button press, $25 \mathrm{~ms}$ corresponding to the delay between EMG activity and button press as recorded separately in one subject with this setup). This result is compatible with a model in
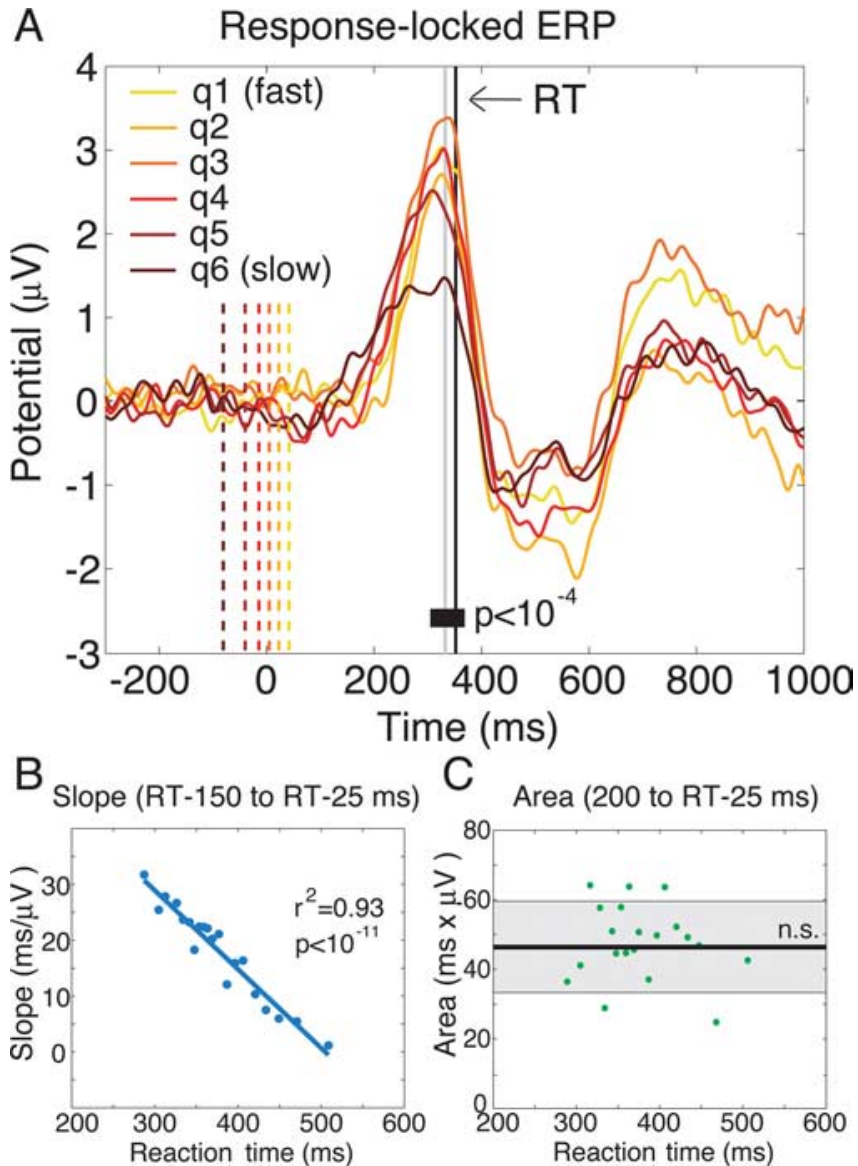

Figure 3. Correlation between reaction time and P3f amplitude. $\boldsymbol{A}$, Response-locked ERP for the right suborbital electrode in six reaction-time quantiles (q1-q6), each summing the same number of trials from each subject. Dashed lines show the quantile-median stimulus onset times; the solid gray line represents the peak of the ERP/RT correlation 25 ms before the recorded button press (visualized at the mean subject-median RT in solid black). The P3f peak preceding the motor response was smaller before longer-RT responses. The black horizontal bar below the ERPs indicate time windows within which linear regression between RT and ERP amplitude in single trials was significant $(p<0.0001)$. $\boldsymbol{B}$, Linear regression on RT of the best-fitting upslope of 20 response-locked RT-quantile ERPs (measured from $150 \mathrm{~ms}$ to $25 \mathrm{~ms}$ before the response). Note the strong linear trend $\left(r^{2}=0.93\right)$. C, Linear regression on RT of the area under the response-locked P3f (in the same time window used in $\boldsymbol{B}$ ). The horizontal black line indicates the mean area, and the shaded region its (less than or equal to $\pm 1 S D$ ) range. The area under the response did not covary with RT ( $p=0.67 ;$ NS).

which the behavioral response is triggered when the area under the ERP (presumably indexing the cumulative amount of underlying neural activity) reaches a threshold. Consistent with this hypothesis, the area under the P3f in the same time range (Fig. $3 C$ ) was not correlated with response time ( $p=0.67, \mathrm{NS})$.

\section{Time-frequency analysis}

Studying correlations between RTs and average ERPs, however, fails to capture the complexity of EEG dynamics in the single trials in which the RT variations occur. A complementary approach to computing correlations between RTs and average ERP dynamics is to study correlations between RT and EEG timefrequency transforms of single trials. Figure 4 shows the mean spectral characteristics of the response-locked suborbital channel data via the event-related spectral perturbation (ERSP) measure of mean power spectral changes time-locked to experimental events (Makeig, 1993). In the channel ERSP, spectral power increased by $\sim 2 \mathrm{~dB}(59 \%)$ at frequencies below $7 \mathrm{~Hz}$. Above $4 \mathrm{~Hz}$, 

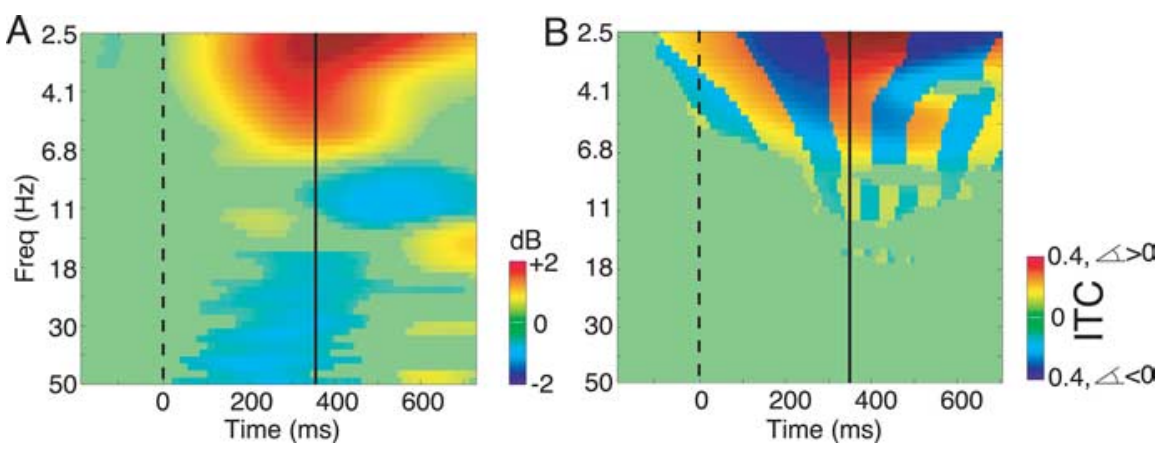

Figure 4. $\quad \boldsymbol{A}$, Grand-mean (15 subjects) ERSP image showing decibel changes in spectral power in response-locked artifactcorrected EEG trials from the right suborbital electrode. Solid line, Actual button press; dashed line, median stimulus onset. $\boldsymbol{B}$, Grand-mean ITC magnitude, measuring phase consistency across single-trial channel data, and phase sign (blue, $<0$; red, $>0$ ). Green areas are nonsignificant (see Materials and Methods).
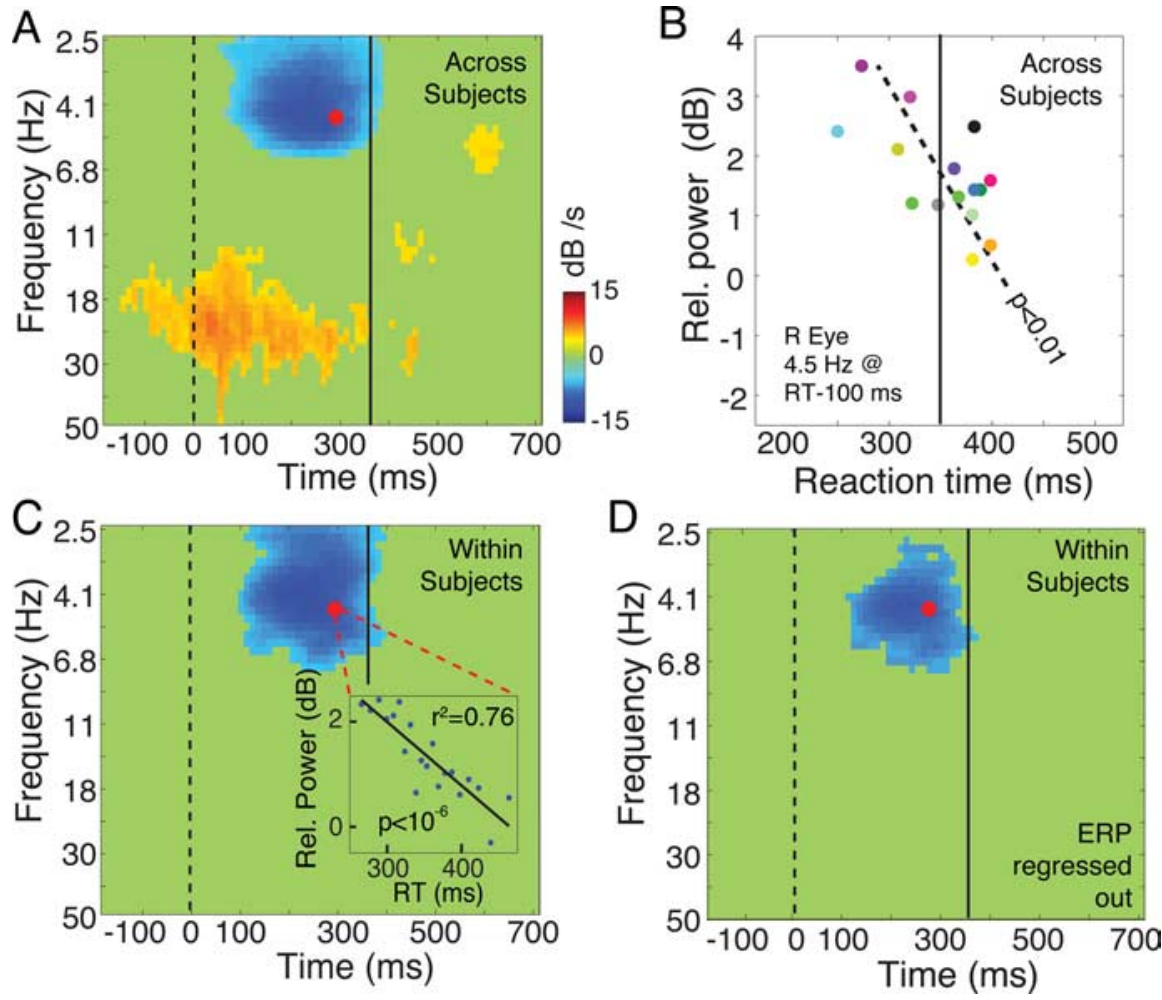

Figure 5. Correlations between reaction time and spectral power. $\boldsymbol{A}$, Time-frequency correlation image showing correlations between absolute RT (in milliseconds) and single-trial EEG log power at the right suborbital electrode, across all trials and subjects. The vertical line is the actual button press (labeled as occurring at the mean subject-median RT, $352 \mathrm{~ms}$ ); the dashed line is median stimulus onset time. In the negatively correlated (blue) region, larger EEG power was associated with faster responses. $\boldsymbol{B}$, Acrosssubjects correlation: dots give median RT of each of the 15 subjects versus mean low-theta log power in a three-cycle timefrequency window centered 100 ms before RT. The linear regression (dashed line) is significant $(p<0.01)$. C, Within-subject correlations, as in $\boldsymbol{A}$, but after subtracting the subject-median RT from each trial RT to minimize effects of subject differences. The red dot indicates the center of a time-frequency region in which larger low-theta log power preceded faster responses (i.e., shorter RTs) in single trials. Inset, Mean low-theta log power in $20 \mathrm{RT}$ quantiles is linearly related to quantile-mean RT $(r=0.87$; $\left.p<10^{-6}\right)$. $\boldsymbol{D}$, Same as $\boldsymbol{C}$ but for low-theta log power remaining in single trials after regressing out the subject-mean ERP from each trial (see Materials and Methods). The maximum correlation is weaker than in $\boldsymbol{C}(\boldsymbol{C}$ and $\boldsymbol{D}$ use the same color scale as $\boldsymbol{A})$, but the same time-frequency correlation pattern remains.

this increase was centered just before the motor response record (Fig. 4A), while below $4 \mathrm{~Hz}$, somewhat after it.

ITC is a measure of the consistency of spectral phase of recorded activity in single trials (Tallon-Baudry et al., 1996; Makeig et al., 2002; Delorme and Makeig, 2004). Below $7 \mathrm{~Hz}$, the ITC image (Fig. $4 B$ ) also exhibits statistically robust though partial $(r<0.4)$ phase locking of the EEG activity to the motor response, this significance remaining in effect, above $4 \mathrm{~Hz}$, for at least two cycles. Thus, in both the ERSP and ITC images, two separate dynamic patterns appear, one below $4 \mathrm{~Hz}$ and another between $\sim 4$ and $6 \mathrm{~Hz}$. A third small, albeit weakly, significant ITC feature appears (Fig. 4B) in the $\alpha$ frequency range (near $10 \mathrm{~Hz}$ ) centered shortly after the subject response. Note that in Figure $5 A$ (see below), this time-frequency feature does not correlate with reaction time in single trials. Thus, the same channel data exhibit at least one other eventrelated dynamic change that precedes the motor response but shows no correlation to reaction time.

\section{Time-frequency regression}

Next, we determined the times and frequencies at which spectral amplitude in single trials at the right suborbital electrode was correlated with RT. To do this, we regressed actual RT on single-trial spectral power and phase estimates computed in three-cycle windows at a grid of time-frequency points (see Materials and Methods) to create a time-frequency regression image (Fig. 5A). At frequencies below $6 \mathrm{~Hz}$, stronger oscillatory activity preceded faster responses (Fig. $5 A$, blue area), whereas between 15 and $30 \mathrm{~Hz}$, stronger oscillatory activity preceded slower responses (Fig. 5A, yellow region).

Next, we asked whether, in general, faster subjects also produced more lowtheta activity before their motor responses. In the time-frequency window with highest log-power/RT correlation (RT - 100 $\mathrm{ms} ; 4.5 \mathrm{~Hz}$ ), circled in Figure $5 A$, the median response times of the 15 subjects were correlated $(p<0.01)$ with subject-mean EEG log power (Fig. 5B). Thus, faster subjects tended to have larger and/or more frequent low-theta complexes peaking in amplitude near a positive peak just before their motor responses.

Next, we subtracted each subject's median RT from each single-trial RT and again computed the time-frequency correlation image. This minimized the effect of across-subject differences, because all subjects now had the same median RT (plotted in Fig. $5 C$ at the group-mean subject-median RT, $352 \mathrm{~ms}$ ). In the RTadjusted correlation image (Fig. 5C), the earlier and higher-frequency correlation region (centered near $20 \mathrm{~Hz}$ in Fig. 5A) disappeared, indicating it arose mainly from between-subject differences. Importantly, the significant low-frequency correlation remained.

Using 20 RT quantiles for each subject, we determined that within subjects, subject-adjusted RT was linearly related to lowtheta $\log$ power $\left(r^{2}=0.76 ; p<10^{-6}\right)$ (Fig. $5 C$, inset). Thus, both 

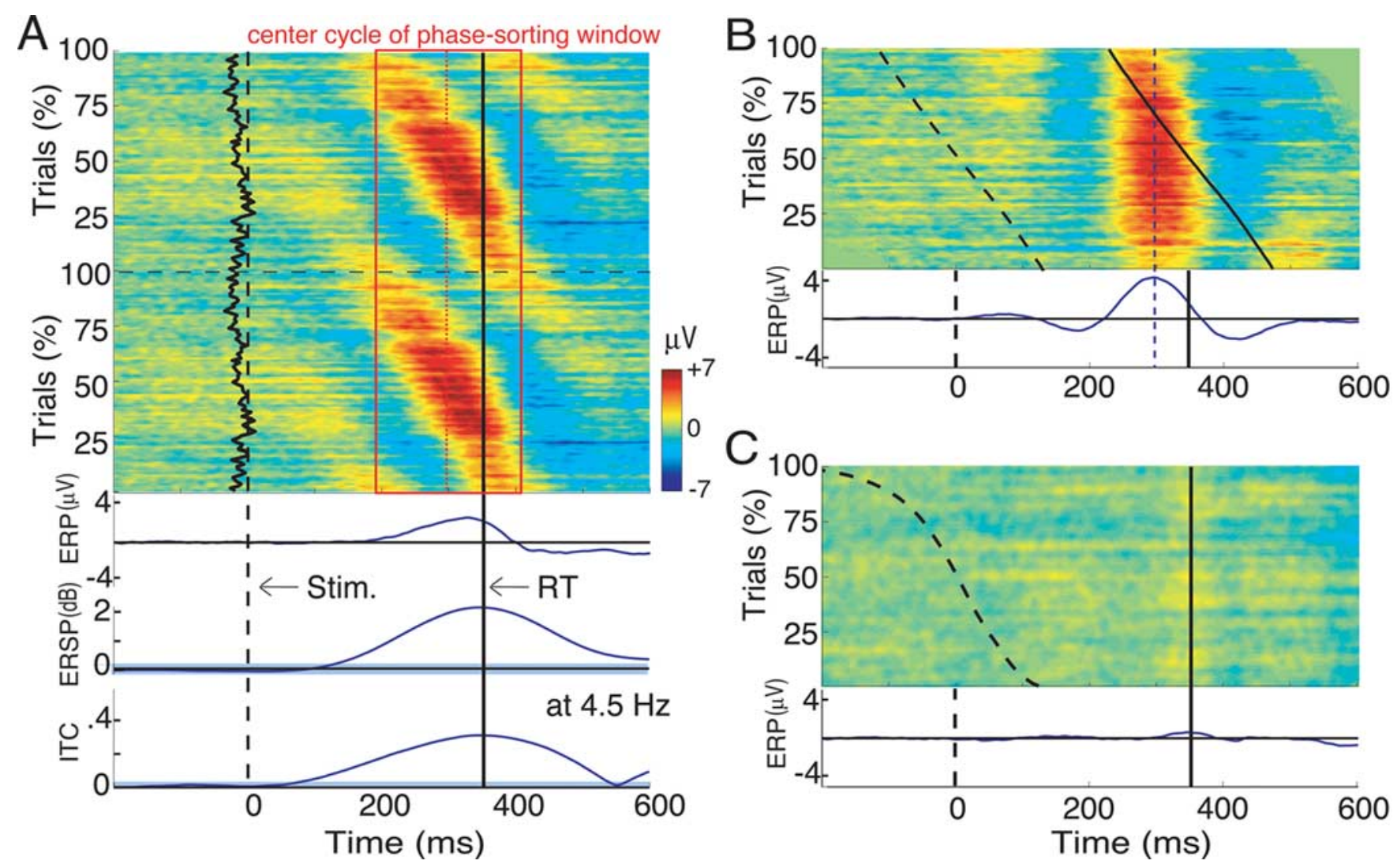

Figure 6. Phase-sorted ERP-image plots showing that the grand-mean ERP arises from partial phase synchronization of a low-theta complex. $\boldsymbol{A}$, Grand-mean ERP-image plot of response-locked trials sorted vertically by phase near $4.5 \mathrm{~Hz}$ in a three-cycle window centered at the grand-mean P3f peak latency, 52 ms before the response, indicated by the red dotted line at the mean subject-median RT, $352 \mathrm{~ms}$. Each trial is shown twice (below, above). Trial images for each subject were smoothed vertically with a 10-trial moving and then grand averaged across subjects. Dotted line, Grand-median stimulus presentation time; thick jagged vertical trace (near time 0), average stimulus onset time for each moving-average trial subset; box, limits of the three-cycle timefrequency window used to compute phase. The three traces below the ERP image show the mean trial ERP (top), ERSP in spectral log power (middle), and ITC. The boundaries of the (thin horizontal) shaded blue areas in the two bottom traces indicate $p<0.01$ significance limits. $\boldsymbol{B}$, The same single trials realigned to low-theta phase in the indicated $(\boldsymbol{A})$ time-frequency window. Black trace, Moving-average RT; dotted trace, moving-average stimulus onset; trace below, grand low-theta phase-locked ERP. $\boldsymbol{C}$, RT-sorted ERP image after regressing out the mean low-theta complex ( $\boldsymbol{B}$ ) from each single trial. The P3fERP peak is now nearly absent.

within and between subjects, larger low-theta EEG power at the suborbital electrode preceded faster motor responses. Note the differences between Figures $4 A$ and $5 A$ : although at this suborbital site low-frequency power was larger, on average, both before and after faster responses (Fig. 4A), only EEG activity before the response was associated with faster responding (Fig. $5 A$ ), and this effect was separate from the subject effect (Fig. 5C).

To determine whether the observed correlations arose only from a subset of trials with relatively high or low theta power, we analyzed the distribution of the correlated low-theta log power across single trials for three RT ranges from the time-frequency RT correlation image with subject mean RT subtracted in Figure $5 C$. The log power histograms (not shown) had a similar shape in all three ranges but were shifted toward higher power preceding faster responses. Thus, the observed correlation (Fig. 5C) was not produced solely by subsets of trials with abnormally high or low spectral power.

Time-frequency decomposition of the response-locked ERP averages themselves demonstrated that low-theta power, in a window centered at the P3f peak, increased by $\sim 2 \mathrm{~dB}$ (59\%; data not shown) compared with the prestimulus baseline activity, similar to the total single-trial low-theta power increase in Figure $4 A$. To test whether the low-theta/RT correlations arose mainly from the correlation between RT and P3f peak amplitude in the response-locked ERP (Fig. 3), we regressed out the subject-mean

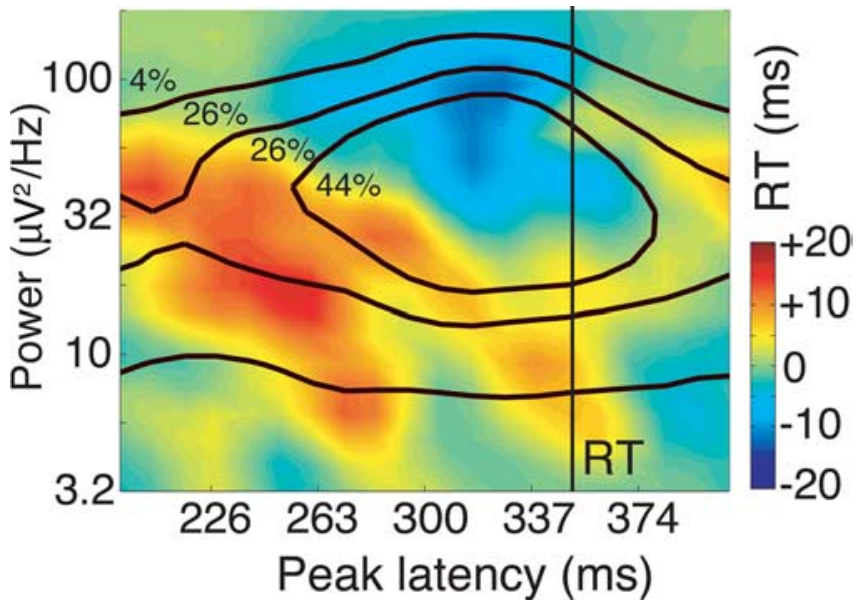

Figure 7. Relative mean reaction time (colors) and reaction time density (level lines) as functions of theta complex peak latency and amplitude. Mean RT deviation (in milliseconds) relative to median RT (color scale) and relative RT density (level lines) are estimated as a function of phase and power at $4.5 \mathrm{~Hz}$ in a three-cycle window centered 50 ms before the button press (indicated for convenience as occurring at the mean subject-median RT, $352 \mathrm{~ms}$ ). Phase indexed by the latency of the positive peak. Level lines indicate relative trial densities. Lowtheta complexes with low amplitudes (bottom), and those not immediately followed by a button press (left), were associated with longer RTs (red), whereas more common highamplitude complexes followed immediately by a button press (top middle) were associated with shorter RTs (blue). 
A

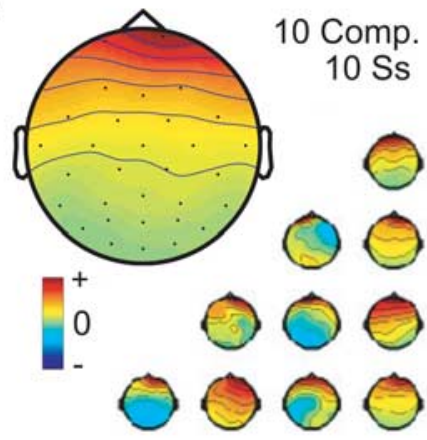

B

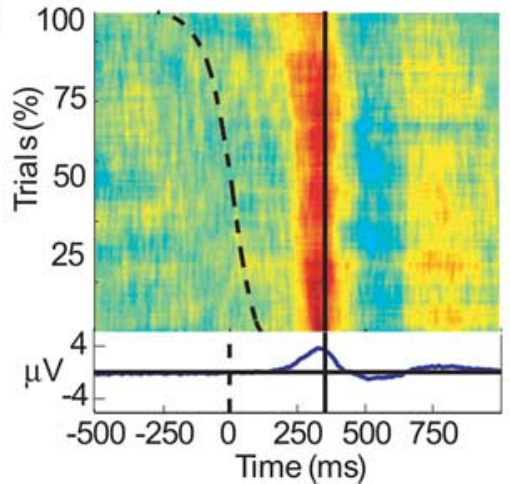

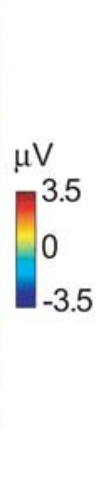

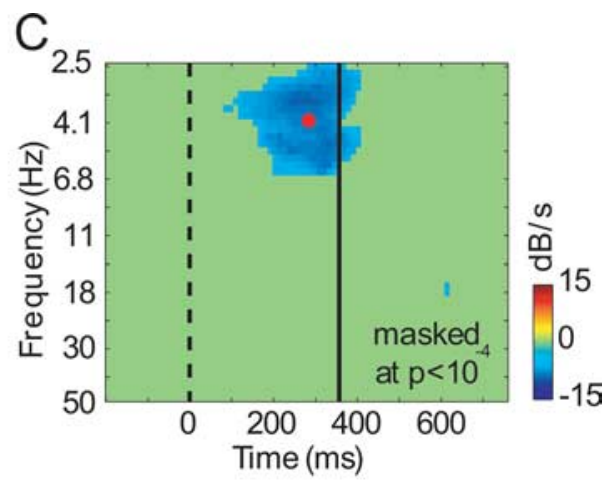

Figure 8. Far-frontal P3f independent component cluster activity. $\boldsymbol{A}$, Mean component cluster scalp map (large), plus individual component maps from 10 subjects (small). $\boldsymbol{B}$, ERP-image plot of RT-sorted component cluster activity. Single-trials for each component were z-normalized by dividing by the SD of the prestimulus component activity. Each color-coded row in the image represents the moving average of 300 normalized data trials aligned to the response time and sorted by its (RT) latency from stimulus onset (dotted line). Larger positive P3f potentials preceding quicker button presses. C, Within-subject correlations between spectral log power and RT in the P3f component cluster. Median RT differences were removed before correlating to minimize the effects of overall subject RT differences.

S1

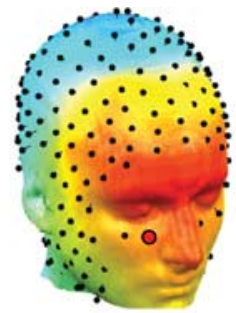

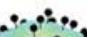

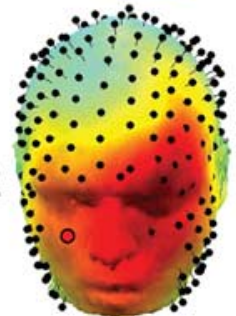

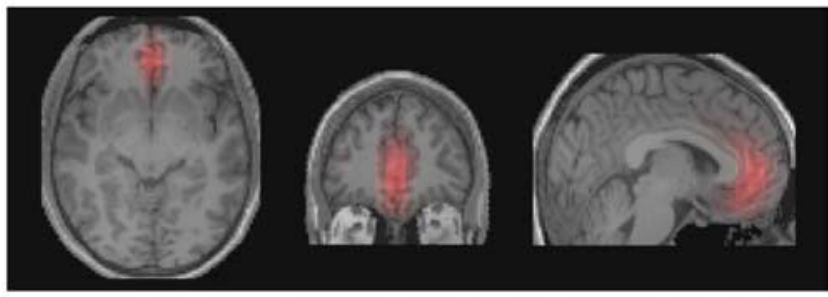

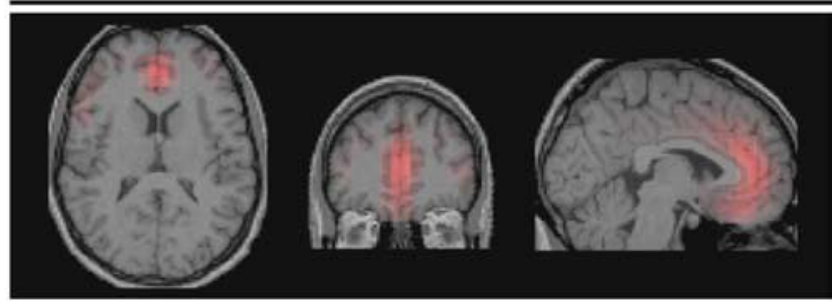

Figure 9. Independent component processes accounting for portions of the premotor P3f positivity in 253-channel data from two subjects. Left, Interpolated component scalp maps on the subject head surface (from anatomic MR images). Black pins indicate the digitized positions of the electrodes; the right suborbital scalp channel location is circled. Right, 3-D distribution of probable component source density under LORETA decomposition.

response-locked ERP from every single trial. To do this, before subtracting the subject response locked ERP to each individual trial, we scaled the ERP (over the whole ERP time range) to best match each single trial individually. The result (Fig. 5D) showed that the portion of the low-theta activity in single trials not phase locked to the button press showed the same pattern of low-theta power/RT correlation as did the phase-locked portion of the single-trial data. Note, however, that the evoked (ERP) and induced (ERP-removed) data portions may not necessarily be functionally distinct.

Phase-sorted single trials

Although the ERP image plots in Figure 2 clearly indicate that the amplitude of the P3f positivity varied from trial to trial, each horizontal row in those images was an average of 150 trials with similar RTs (10 trials times 15 subjects) and thus could hide the existence of a considerable portion of the data in trials in which the latency of the premotor excursion, or the phase of the premotor low-theta activity may have differed from the mean P3f latency in the ERP. To attempt to better understand the relationship between the ERP/RT and the low-theta/RT correlations, we next analyzed single-trial low-theta power as a function of its phase relative to the button press.

Average ERPs ignore data features that are not both time locked and phase locked to the time-locking events (Makeig, 1993). Inspection of average ERPs may thus foster the incorrect assumption that peaks at the same latencies as in the average ERP must be evoked by near-identical time-locking events in all or nearly all the single trials, as is the case, for example, for lateral occipital responses evoked by onsets of foveal visual stimuli (Makeig et al., 2004a). Figure 6A plots the same single-trial data as Figure $2 B$, but now sorted not by RT but by phase in a three-cycle, $4.5-\mathrm{Hz}$ time/frequency window centered $52 \mathrm{~ms}$ before button press (on average $300 \mathrm{~ms}$ after stimulus onset, because the grand-median RT was 352 $\mathrm{ms})$. The analysis frequency was at or near the peak frequency of the average ERP in this time window. Because phase is a circular measure, for visual clarity, the phase-sorted ERP image plot shows the phase-sorted data trials twice (i.e., through two revolutions around the phase circle, from 0 to $4 \pi$ ). Detailed inspection of the phase-sorted data revealed that the features of Figure $6 \mathrm{~A}$ were not dominated by data from a few of the subjects, over half the subjects contributing near equally to each row of the data image.

\section{Low-theta complex}

The phase-sorted ERP image of the response-locked epochs (Fig. 6 A) shows that, whereas the mean P3f positivity peaks $\sim 50 \mathrm{~ms}$ before the recorded button press in many trials, in many other trials, the principal theta-band positivity occurs either earlier or later than this. This phase sorting of the single-trial data demonstrates that an approximate 1.5 cycle (negative-positive-negative) low-theta complex appears in most trials closely preceding the motor response (Fig. $6 B$ ).

In the RT-sorted ERP-image panels shown in Figure $2 B$, however, this activity pattern is hidden, particularly the weak negativity that consistently precedes the P3f peak (Fig. 6B). Trials at which the phase of the low-theta activity is farthest from the mean 
P3f pattern appear to have smaller low-theta activity, whereas the largest mean low-theta complex is associated with a $\mathrm{P} 3 \mathrm{f}$ positivity at and near the ERP peak latency. Note also in Figure $6 \mathrm{~A}$ that trials with an early P3f peak have slower mean RT. These trends in the variability of the single data trials are hidden both by the RT-sorted ERP image (Fig. 2 B) and by the average ERP (Fig. 3 ). Finally, Figure $6 C$ shows that regressing out the low-theta complex (see Materials and Methods) from the data removed most of the ERP.

\section{Combined effects of low-theta amplitude and phase on RT}

Figures 5 and 6 indicate that subject RT in these experiments was related to the power and phase of a low-theta complex usually peaking before the subject button press at the subocular channel. To assess the relative and combined effects of phase and power of the P3f low-theta complex, we computed smoothed 2-D histograms of relative trial density and of localmean RT as a function of low-theta power and phase relative to the button press. Results (Fig. 7) confirm that quicker responses are associated with larger low-theta complexes peaking near the mean P3f latency (blue area), whereas low-theta complexes with a positive peak near to a half-cycle $(111 \mathrm{~ms})$ before the button press are associated with RTs up to $40 \mathrm{~ms}$ slower than average (red area).

\section{Independent component analysis}

Analyzing the activity recorded at the single suborbital electrode provided a good starting point for studying correlations between EEG activity and RT. However, each single-channel recording typically sums EEG activities originating in many parts of the cortex. Thus, we next attempted to locate the EEG sources with contributions to the scalp EEG that best accounted for the RT correlations observed at the suborbital electrode. To do this, we applied ICA to $\sim 500$ concatenated 31-channel, $1 \mathrm{~s}$ trials time locked to target presentations for each subject, using extended infomax ICA (see Materials and Methods), and clustered the resulting independent components across subjects on the basis of similarities in scalp maps and activity spectra. This gave 15 component clusters. We focused here on one cluster we had previously dubbed P3f, because it accounted for most of the preresponse P3f peak in the ERP at the same suborbital channel (Makeig et al., 1999a). Other component clusters, previously described in more detail, accounted for both preresponse and postresponse EEG activity projecting most strongly to the central midline, frontal midline, parietal, left and right central, and posterior scalp areas, respectively (Makeig et al., 2004b).

The scalp maps of the P3f cluster components (Fig. 8A) showed activity projecting mostly to the suborbital and frontal electrodes. Ten of the 15 subjects contributed components to this cluster, which accounted for $88 \%( \pm 13 \% \mathrm{SD})$ of the early portion of the grand mean stimulus-locked P3f peak (200-300 ms) for these subjects. Because this component topography resembled that of eye artifact, we investigated each subject component activity to ensure that it did not contain activity consistent with eye movements (supplemental Fig. 10, available at www. jneurosci.org as supplemental material).

As with the suborbital channel data, the component cluster ERP image plot (Fig. 8 B) suggests a correlation between RT and the far-frontal component cluster positivity preceding the button presses. Again, the higher the component P3f peak, the quicker the button press $\left(p<10^{-4}\right)$. We also computed correlations between single-trial RT and log power between 2.5 and $50 \mathrm{~Hz}$, after subtracting subject median RT from each trial RT to mini- mize effects arising from subject RT differences (Fig. $8 C$ ). The time-frequency correlation image before subtracting median reaction time (data not shown) resembled Figure $5 C$. When we also regressed out the grand average ERP from each trial (data not shown), the same low-theta correlation pattern was preserved, indicating that most of the correlations between low-theta log power and RT for the component cluster were again not generated by amplitude modulation of the ERP pattern in single trials.

\section{Source localization}

Equivalent dipole source modeling applied to these relatively sparsely sampled 31-channel data could only provide a general idea of the inferior frontal source locations of the P3f cluster components. Using a four-shell spherical model with default conductance and sphere radius parameters [BESA 3.0 (Scherg and Cramon, 1985)], we obtained single-dipole fits with low residual variance (mean, $6.2 \pm 5.1 \%$ ) clustered in the medial inferior head model, with a $3-\mathrm{D} S \mathrm{SD}<2 \mathrm{~cm}$. However, some of the equivalent component dipoles were located below the brain volume (data not shown).

\section{High-density recordings}

To attempt to better determine the location of the independent components accounting for periocular P3f activity, we recorded new EEG data for the same task from two subjects using 253 electrodes covering most of the head surface and derived 150 maximally independent data components. For both subjects, ICA identified not one but two nonartifactual independent components that produced a clear frontal-positive premotor response ERP peak, as well as a low-theta power increase. Here, we present the component from each subject that contributed most to the activity at the right peri-occular channel. We localized their probable source in each subject's anatomic MR image using LORETA (Pascual-Marqui et al., 1994) (see Materials and Methods) to within and/or near bilateral frontopolar and ventral cingulate cortex (Fig. 9). These components accounted for 30 and 13\%, respectively, of the whole right suborbital channel activity.

For these two subjects, the response-locked P3f component activity in single trials at the latency of the average responselocked ERP peak was correlated with trial RT $(p<0.001$, in both cases). Although in longer RT trials the component for subject S1 remained time locked to stimulus onset rather than to the button press (supplemental Fig. 11, available at www.jneurosci.org as supplemental material), correlations between RT and the response-locked P3f component slope in the $200 \mathrm{~ms}$ to $(\mathrm{RT}-25)$ ms time range in single trials were also significant $(p<0.001$ and $p<0.002$, respectively). Similar results were obtained for the whole suborbital channel signals after eye and muscle artifact removal (RT/P3f-peak potential correlations, $p<0.01, p<$ 0.001 ; RT/P3f-upslope correlations, $p<0.001$, NS). As with the 31-channel data, $50 \mathrm{~ms}$ before the subject response, the ensuing RT was correlated with theta power (supplemental Fig. 11, available at www.jneurosci.org as supplemental material $)(p<0.002$ at $5 \mathrm{~Hz} ; p<0.05$ at $4.5 \mathrm{~Hz}$ ).

\section{Discussion}

First, we have shown in more detail that the latencies of individual speeded responses to infrequent visual target events in a visual-spatial selective attention experiment were correlated with the size, in the single trials, of a preceding $\mathrm{P} 3 \mathrm{f}$ ramping positivity (Potts et al., 1998; Makeig et al., 1999b) projecting most strongly to the forehead and upper face (Fig. 9). A similar or identical positivity, termed P2a, has been reported by Potts et al. (1998). Next, we showed that faster motor responses tended to follow 
larger P3f peaks. Third, we demonstrated that the ramping P3f positivity could also be modeled as a triphasic low theta $(4.5 \mathrm{~Hz})$ complex weakly phase locked to the button presses. Overall, larger low-theta complexes, including a larger P3f-contributing positivity, preceded quicker motor responses, both within and across subjects.

Because no other channel in our 31-channel electrode montage was within the main $\mathrm{P} 3 \mathrm{f}$ projection region (compare Figs. $2 \mathrm{~A}$ and 9), we first used data from a right subocular electrode to establish the above facts. By removing artifacts arising from eye movements using ICA (Jung et al., 2000), we were able to extract clear information about far-frontal event-related EEG brain dynamics from the periocular channel data. Next, we showed that ICA separated much of the P3f activity in the 31-channel data sets into an independent component. In two 253-channel data sets, quite similar independent component processes appeared and were modeled by LORETA as most likely generated in ventromedial prefrontal cortex (BA 32/10).

\section{P3f and low theta}

Time-frequency analyses showed that the positive P3f peak in the average ERP time locked to the button presses in our experiments was principally produced by triphasic (negativepositive-negative), circa $4.5 \mathrm{~Hz}$ low-theta complexes. In single trials, both the amplitude and phase of these low-theta complexes, preceding the button presses, were partially correlated with RT (Figs. 4-7). ERP energy at frequencies above the theta band, although relatively small (Fig. 6C), was clearly present (Figs. $3 A, 4 B$ ). Thus, the P3f peak cannot be described as a purely low-theta process. Rather, a low-theta complex can be said to underlie the P3f peak in the following sense: regressing out the response-locked ERP from each trial failed to remove the low-theta/RT correlation, whereas regressing out the lowtheta complex from each trial not only destroyed the correlation between the remaining ERP and RT, but nearly removed the response-locked ERP altogether.

It is common to think of ERP activity as reflecting consistent polarity or phase locking of the single-trial EEG separately at each indicated latency relative to the time-locking events. However, similar to the pattern exhibited by the slightly later theta-band response dynamics appearing at other cortical locations in our 31-channel data (Makeig et al., 2004b), phase locking of triphasic $(300 \mathrm{~ms})$ low-theta complexes contributing to the P3f, although statistically significant, was far from complete. Therefore, the waveforms of the response-locked ERP (Fig. $2 B$ ) and of the recovered partially response-locked theta complexes (Fig. $6 \mathrm{~B}$ ) appear quite different. It must be no coincidence that the premotor response ERP average in these experiments resembles a linear upwards ramp, whereas thetaphase trial sorting indicates that variably timed and powered low-theta complexes dominate the single trial data. Accounting for both these facts in a single generative model is a clear challenge to EEG modeling. The nature and function of the phase or latency variability of the P3f (and later) theta complexes, relative to the motor command, might also be further explored by studying the exact conditions or contexts in which the response differences occur (Onton et al., 2005).

\section{Neuronal correlates}

The very tight observed correlation between reaction time and the steepness of the upward P3f slope (Fig. 3B) is reminiscent of the demonstration by Hanes et al. (1995) of monotonic ramping up of firing rates of neurons in the frontal eye fields of monkeys up to the release of cued saccades. Amano et al. (2006) also showed correlations of reaction time with average and single-trial magnetoencephalographic signal above occipital cortex and to a lesser extend over frontal cortex. This indicates our RT correlation results in the frontal cortex may be related to a general mechanism for releasing speeded button presses that is not particular to the task we used. Because our time-frequency analyses showed that the linear ramp in the P3f peak (Fig. 3B) masked a triphasic theta-band complex, it appears possible that similar results obtained by response-locked averaging of spike occurrences might similarly mask complex or partly oscillatory structures of spike timings and/or associated local field potentials, effects that may well have been hidden by standard spike and field averaging methods.

\section{P3f localization}

In at least 10 of the 15 subjects, ICA decomposition of the concatenated 31-channel data trials found an independent component process that primarily accounted for the P3f peak in the response-locked ERP. This relatively sparse recording montage did not allow sure equivalent-dipole localization of inferior frontal sources. Application of a low-resolution inverse model, LORETA, to pairs of similar independent components recovered from data in two separate experiments using a 253-channel electrode montage modeled the $\mathrm{P} 3 \mathrm{f}$-generating processes as most likely located in bilateral frontopolar and/or ventral cingulate and/or paracingulate (BA32/10) cortex (Fig. 9). LORETA is currently considered to be an unbiased method for localizing EEG and ERP sources (Yao and Dewald, 2005), but it only provides probabilistic localization information. As summarized below, a number of hemodynamic imaging results appear compatible with the most probable source location as determined by LORETA, although other nearby source locations cannot be excluded.

In "Go/NoGo" task studies, a region of cingulate cortex similar to that shown in Figure 9 has been shown to be involved in response inhibition (Booth et al., 2003; Jaffard et al., 2006). Lesions in medial orbitofrontal cortex are also associated with loss of behavioral inhibition (Cato et al., 2004). Similarly, attention deficit disorder patients have been shown to exhibit abnormal frontal release from inhibition (Booth et al., 2005). Thus, our EEG results suggest that preceding faster speeded responses, medial orbitofrontal cortex undergoes an early release from response inhibition accompanied by a low-theta complex.

\section{$P 3 f$, response speed, attention, expectation, and reward}

The published functional imaging results summarized above appear compatible both with the P3f ERP and low-theta results we presented here, as well as with the results of Martin and Potts (2004), who reported that the highly similar peak in ERPs time locked to feedback signals in a simple gambling task was pronounced only after visual stimuli that cued an unexpected reward. For our subjects, the attended but relatively infrequent and unpredictably timed target stimulus presentations may be said to have been rewarding events (e.g., sudden opportunities to obtain the satisfaction of producing the timely and accurate responses requested by the experimenter). Cingulate activations near the frontal pole, localization of our P3f-generating processes (Fig. 9), have been reported in several recent fMRI experiments involving responses to unpredictably timed targets or other rewarding events. Knutson et al. (2003) have shown that medial prefrontal cortex (BA32/10) is activated to quick, winning button presses. A similar locus of activity has been associated with quick task- 
relevant shifts in attention to target cues (Wager et al., 2005). Finally, Laurienti et al. (2003) have shown a specific association between BA32/10 activation and quicker manual reaction times in response to coherent (compared with incoherent) audiovisual stimuli. These functional imaging results firmly associate activity in rostral anterior cingulate and medial frontopolar cortex with awareness of and facilitated motivated behavioral in response to target stimuli (Mayr, 2004).

\section{$R T$ and EEG}

Previously, we have shown that the P3f low-theta complexes in the present data were followed by partially phase-coherent EEG theta complexes in otherwise independent EEG source activities in or near dorsal anterior cingulate, pre- supplementary motor area, and (left) somatomotor cortex (Makeig et al., 2004b). A statistical test of transient coherence between these other component clusters revealed that after the button press, low-theta (P3a) complexes at those sites were first partially coherent with each other and then became weakly coherent with the P3f low-theta activity. This is compatible with observed roles of ventral and dorsal anterior cingulate cortex (during P3f and P3a) in anticipating and dealing with the consequences of actions and events.

Our EEG results and related fMRI results discussed above appear compatible with the theory of Wundt (1913), who suggested that fast responders in simple speeded-response paradigms are able to respond reflexively, without reflectively (e.g., consciously), confirming the nature of the response-cueing event. If his theory was accurate, our results might suggest how different neural activity patterns are connected to subject differences in "conscious" experience during rapid responding, correlated with activity differences in or near medial orbitofrontal and/or cingulate cortex.

\section{References}

Amano K, Goda N, Nishida S, Ejima Y, Takeda T, Ohtani Y (2006) Estimation of the timing of human visual perception from magnetoencephalography. J Neurosci 26:3981-3991.

Ashburner J, Friston KJ (2003) Spatial normalization using basis functions. In: Human brain function, Chap 33, Ed 2 (Frackowiak RSJ, Frith C, Dolan R, Friston KJ, Price CJ, Zeki S, Ashburner J, Penny WD, eds), pp 655-672. San Diego: Academic.

Bahramali H, Gordon E, Li WM, Rennie C, Wright J, Meares R (1998) Fast and slow reaction times and associated ERPs in patients with schizophrenia and controls. Int J Neurosci 95:155-165.

Bell AJ, Sejnowski TJ (1995) An information-maximization approach to blind separation and blind deconvolution. Neural Comput 7:1129-1159.

Bonferroni CE (1950) Sulle medie multiple di potenze. Bollettino dell'Unione Matematica Italiana, 53:267-270.

Booth JR, Burman DD, Meyer JR, Lei Z, Trommer BL, Davenport ND, Li W, Parrish TB, Gitelman DR, Mesulam MM (2003) Neural development of selective attention and response inhibition. NeuroImage 20:737-751.

Booth JR, Burman DD, Meyer JR, Lei Z, Trommer BL, Davenport ND, Li W, Parrish TB, Gitelman DR, Mesulam MM (2005) Larger deficits in brain networks for response inhibition than for visual selective attention in attention deficit hyperactivity disorder (ADHD). J Child Psychol Psychiatry $46: 94-111$.

Cato MA, Delis DC, Abildskov TJ, Bigler E (2004) Assessing the elusive cognitive deficits associated with ventromedial prefrontal damage: a case of a modern-day Phineas Gage. J Int Neuropsychol Soc 10:453-465.

Delorme A, Makeig S (2004) EEGLAB: an open source toolbox for analysis of single-trial EEG dynamics including independent component analysis. J Neurosci Methods 134:9-21.

Draper N, Smith H (1998) Applied regression analysis, Ed 3. New York: Wiley.

Gonzalez Andino SL, Michel CM, Thut G, Landis T, Grave de Peralta R (2005) Prediction of response speed by anticipatory high-frequency (gamma band) oscillations in the human brain. Hum Brain Mapp $24: 50-58$
Grossman A, Morlet J (1985) Decomposition of functions into wavelets of constant shape, and related transforms. In: Mathematics+Physics, lectures on recent results, Vol 1 (Streit L, ed). Singapore: World Scientific.

Hanes DP, Thompson KG, Schall JD (1995) Relationship of presaccadic activity in frontal eye field and supplementary eye field to saccade initiation in macaque: poisson spike train analysis. Exp Brain Res 103:85-96.

Hohnsbein J, Falkenstein M, Hoormann J, Blanke L (1991) Effects of crossmodal divided attention on late ERP components. I. Simple and choice reaction tasks. Electroencephalogr Clin Neurophysiol 78:438-446.

Jaffard M, Benraiss M, Longcamp M, Velay J, Boulinguez P (2006) Classical cueing studies are biased in their methods: an event-related fMRI evidence. NeuroImage 31:S50.

Jokeit H, Makeig S (1994) Different event-related patterns of gamma-band power in brain waves of fast- and slow-reacting subjects. Proc Natl Acad Sci USA 91:6339-6343.

Jung TP, Makeig S, Humphries C, Lee TW, McKeown MJ, Iragui V, Sejnowski TJ (2000) Removing electroencephalographic artifacts by blind source separation. Psychophysiology 37:163-178.

Kida T, Nishihira Y, Hatta A, Wasaka T, Nakata H, Sakamoto M, Nakajima T (2003) Changes in the somatosensory N250 and P300 by the variation of reaction time. Eur J Appl Physiol 89:326-330.

Kilner J, Bott L, Posada A (2005) Modulations in the degree of synchronization during ongoing oscillatory activity in the human brain. Eur J Neurosci 21:2547-2554.

Klopp J, Marinkovic K, Chauvel P, Nenov V, Halgren E (2000) Early widespread cortical distribution of coherent fusiform face selective activity. Hum Brain Mapp 11:286-293.

Knutson B, Fong GW, Bennett SM, Adams CM, Hommer D (2003) A region of mesial prefrontal cortex tracks monetarily rewarding outcomes: characterization with rapid event-related fMRI. NeuroImage 18:263-272.

Laurienti PJ, Wallace MT, Maldjian JA, Susi CM, Stein BE, Burdette JH (2003) Cross-modal sensory processing in the anterior cingulate and medial prefrontal cortices. Hum Brain Mapp 19:213-223.

Lee TW, Girolami M, Bell AJ, Sejnowski TJ (2000) A unifying informationtheoretic framework for independent component analysis. Comput Math Appl 31:1-21.

Liu HL, Liao WT, Fang SY, Chu TC, Tan LH (2004) Correlation between temporal response of fMRI and fast reaction time in a language task. Magn Res Imag 22:451-455.

Luce D (1986) Response times: their role in inferring elementary mental organization. New York: Oxford UP.

Makeig S (1993) Auditory event-related dynamics of the EEG spectrum and effects of exposure to tones. Electroencephalogr Clin Neurophysiol 86:283-293.

Makeig S, Bell AJ, Jung TP, Sejnowski TJ (1996) Independent component analysis of electroencephalographic data. In: Advances in neural information processing systems, Vol VIII (Touretzky D, Mozer M, Hasselmo M, eds), pp 145-151, Cambridge, MA: MIT.

Makeig S, Jung TP, Bell AJ, Ghahremani D, Sejnowski TJ (1997) Blind separation of auditory event-related brain responses into independent components. Proc Natl Acad Sci USA 94:10979-10984.

Makeig S, Westerfield M, Townsend J, Jung TP, Courchesne E, Sejnowski TJ (1999a) Functionally independent components of early event-related potentials in a visual spatial attention task. Philos Trans R Soc Lond B Biol Sci 354:1135-1144.

Makeig S, Westerfield M, Jung TP, Covington J, Townsend J, Sejnowski TJ, Courchesne E (1999b) Functionally independent components of the late positive event-related potential during visual spatial attention. J Neurosci 19:2665-2680.

Makeig S, Westerfield M, Jung TP, Enghoff S, Townsend J, Courchesne E, Sejnowski TJ (2002) Dynamic brain sources of visual evoked responses. Science 295:690-694.

Makeig S, Debener S, Onton J, Delorme A (2004a) Mining event-related brain dynamics. Trends Cogn Sci 8:204-210.

Makeig S, Delorme A, Westerfield M, Jung TP, Townsend J, Courchesne E, Sejnowski TJ (2004b) Electroencephalographic brain dynamics following manually responded visual targets. PLoS Biol 2:747-762.

Martin LE, Potts GF (2004) Reward sensitivity in impulsivity. NeuroReport 15:1519-1522.

Mayr U (2004) Conflict, consciousness, and control. Trends Cogn Sci 8:145-148.

Mesulam MM, Nobre AC, Kim YH, Parrish TB, Gitelman DR (2001) Het- 
erogeneity of cingulate contributions to spatial attention. NeuroImage 13:1065-1072.

Naito E, Kinomura S, Geyer S, Kawashima R, Roland PE, Zilles K (2000) Fast reaction to different sensory modalities activates common fields in the motor areas, but the anterior cingulate cortex is involved in the speed of reaction. J Neurophysiol 83:1701-1709.

Neville HJ, Lawson D (1987) Attention to central and peripheral visual space in a movement detection task: an event-related potential and behavioral study. II. Congenitally deaf adults. Brain Res 405:268-283.

Nichols TE, Holmes AP (2002) Nonparametric permutation tests for functional neuroimaging: a primer with examples. Hum Brain Mapp 15:1-25.

Onton J, Delorme A, Makeig S (2005) Frontal midline EEG dynamics during working memory. NeuroImage 27:341-356.

Pascual-Marqui R, Michel C, Lehmann D (1994) Low resolution electromagnetic tomography: a new method for localizing electrical activity in the brain. Int J Psychophysiol 18:49-65.

Potts GF, Dien J, Hartry-Speiser AL, McDougal LM, Tucker DM (1998) Dense sensor array topography of the event-related potential to taskrelevant auditory stimuli. Electroencephalogr Clin Neurophysiol 106:444-456.

Proverbio AM, Zani A, Gazzaniga MS, Mangun GR (1994) ERP and RT signs of a rightward bias for spatial orienting in a split-brain patient. NeuroReport 5:2457-2461.

Scherg M, Cramon DV (1985) Two bilateral sources of the late AEP as identified by a spatio-temporal dipole model. Electroencephalogr Clin Neurophysiol 62:32-44.

Schoffelen JM, Oostenveld R, Fries P (2005) Neuronal coherence as a mechanism of effective corticospinal interaction. Science 308:111-113.

Tallon-Baudry C, Bertrand O, Delpuech C, Pernier J (1996) Stimulus spec- ificity of phase-locked and non-phase-locked $40 \mathrm{~Hz}$ visual responses in human. J Neurosci 16:4240-4249.

Tanji K, Suzuki K, Delorme A, Shamoto H, Nakasato N (2005) Highfrequency $\gamma$-band activity in the basal temporal cortex during picturenaming and lexical-decision tasks. J Neurosci 25:3287-3293.

Tenke CE, Kayser J, Fong R, Leite P, Towey JP, Bruder GE (1998) Responseand stimulus-related ERP asymmetries in a tonal oddball task: a Laplacian analysis. Brain Topogr 10:201-210.

Townsend J, Harris NS, Courchesne E (1996) Visual attention abnormalities in autism: delayed orienting to location. J Int Neuropsychol Soc 2:541-550

Travis F (1998) Cortical and cognitive development in 4th, 8th and 12th grade students. The contribution of speed of processing and executive functioning to cognitive development. Biol Psychol 48:37-56.

Ullsperger M, von Cramon DY (2003) Error monitoring using external feedback: specific roles of the habenular complex, the reward system, and the cingulate motor area revealed by functional magnetic resonance imaging. J Neurosci 23:4308-4314.

Wager TD, Jonides J, Smith EE, Nichols TE (2005) Toward a taxonomy of attention shifting: individual differences in fMRI during multiple shift types. Cogn Affect Behav Neurosci 5:127-143.

Womelsdorf T, Fries P, Mitra PP, Desimone R (2006) Gamma-band synchronization in visual cortex predicts speed of change detection. Nature 439:733-736.

Wundt W (1913) Grundriss der Psychologie. Leipzig, Germany: Engelmann.

Yao J, Dewald JP (2005) Evaluation of different cortical source localization methods using simulated and experimental EEG data. NeuroImage 25: 369-382. 